\title{
A Rare Case of Shingles \& Pneumonia Vaccine that led a Healthy Person to Mechanical Ventilator \& Tracheostomy Tube due to Progressive GBS Complicated with Rhabdomyolysis
}

Sajid Mahmood ${ }^{1 *}$, Aminur Rehman Nadeem ${ }^{2}$, Amandeep Dhillon ${ }^{3}$, Karen Carvajal ${ }^{4}$, Mahwash Siddiqui ${ }^{5}$, Sana Chaudhary ${ }^{6}$, Raghda Al-Anbari ${ }^{7}$, Ali Al-Khazaali ${ }^{8}$, Zaid Abood ${ }^{9}$ and Waseem Ahmed ${ }^{10}$

Department of Internal Medicine, Rosalind Franklin University of Medicine and Science, USA

Received: February 01, 2018; Published: February 20, 2018

*Corresponding author: Sajid Mahmood, Department of Internal Medicine, Rosalind Franklin University of Medicine and Science, Illinois, USA, Tel: 3123859432; Email: drsajid@live.com

\begin{abstract}
Background: Guillain-Barre Syndrome (GBS) is an acute polyneuropathy condition which has been reported following vaccinations [1]. We describe a rare case of post-immunization GBS complicated with acute rhabdomyolysis.

Case description: A 71-year-old male presented with progressive weakness of lower and upper extremities, fall, dyspnea started after eleven days of receiving herpes zoster and pneumococcal vaccines. The patient exhibited increased work of breath which led to immediate intubation and later also underwent tracheostomy procedure. Based on clinical presentation, initial diagnostic work up and neurology consultation, diagnosis of GBS was considered and patient received IVIG. During inpatient care serum cpk and urine myoglobin were reported 7634 and 12700 respectively showing development of acute rhabdomyolysis. Patient remained under care of multidisciplinary teams and condition started improving within 4-6 weeks. Later, he was extubated, tracheostomy tube was removed, cpk trended down to normal and patient was transferred to rehabilitation center for continuing physical therapy.

Conclusion: Our patient developed GBS following immunization and later complicated with rhabdomyolysis. This case emphasizes the need to consider GBS in any patient who presents with weakness and muscle pain following vaccinations especially shingles and pneumonia as in our case. It also focuses to closely monitor cpk levels to avoid development of fatal rhabdomyolysis.

Keywords: Vaccination; GBS; Rhabdomyolysis; Herpes zoster; Ventilator

Abbreviations: GBS: Guillain-Barre Syndrome; CPK: Creatinine phosphokinase; HZV: Herpez Zoster Virus; PCV: Pneumococcal conjugate vaccine (PCV); IVIG: Immune Globulin; EMG: Electromyography; CT: Computed Tomography; MRI: Magnetic Resonance Imaging; HTN: Hypertension; COPD: Chronic Obstructive Pulmonary Disease; BP: Blood Pressure; ICU: Intensive Care Unit; CSF: Cerebrospinal Fluid; WBC: White Blood Cell
\end{abstract}

\section{Introduction}

Guillain-Barre syndrome is an acute polyneuropathy condition, which may occur after immunization with live vaccines or infection with Campylobacter jejuni [2]. It is an ascending paralysis which starts from lower extremities and further progresses upwards and in late stages is accompanied by involvement of the respiratory muscles. GBS in rare circumstances is accompanied by Rhabdomyolysis due to muscle damage which if not treated adequately can result in acute renal failure and rarely death. Mild elevation in serum creatine kinase (CK) level have been seen in early stage of GBS, but marked elevation of CK in GBS is an extremely rare finding [3]. We present the case of GBS following immunization with marked increased in serum CPK and myoglobin levels.

\section{Objective}

The objective of this case report is to highlight the significance of considering this rare entity and related complications like pneumonia, pulmonary embolism, respiratory insufficiency, cardiac arrest [4] and rhabdomyolysis especially following routine immunizations, due to its lasting crumbling impact on quality of life for years after diagnosis.

\section{Case Report}

A 71-year-old male with past medical history of HTN, COPD presented with progressive weakness of lower and upper extremities, fall and dyspnea. His symptoms started since three days and were getting worse each day. He has 
received shingles and pneumonia vaccines eleven days ago. He described the weakness as tightness in his legs and reported more significantly diminished strength in his lower extremities than upper extremities. On physical examination patient was a febrile, stable BP, hyporeflexia with total paralysis of both upper and lower extremities. There were no meningeal signs, no facial palsy or hearing loss.

The patient exhibited increased work of breath which led to intubation for mechanical ventilation support in ICU. Based on history, clinical presentation, elevated CSF proteins with normal WBC count, electro radiologic diagnostic work up including EMG, CT head, MRI brain and neurology consultation, diagnosis of GBS was considered and patient received IVIG. The patient underwent a tracheostomy due to noted vocal cord paralysis and oropharyngeal weakness. Subsequently, patient also suffered of aspiration pneumonia and new onset a trial fibrillation. Dark color urinary drainage was noted in catheter bag. Although statins were on hold but serum cpk and urine myoglobin were reported 7634 and 12700 respectively showing development of acute rhabdomyolysis.

Serum potassium and creatinine remained within normal range. He was started on broad spectrum antibiotic coverage and intravenous fluids under care of multidisciplinary teams. Subsequently, patient's condition started improving within four weeks. Later, he was extubated, tracheostomy tube was removed, cpk trended down to normal and patient was transferred to rehabilitation center for continuing physical therapy.

\section{Discussion}

Guillain-Barre Syndrome is an ascending polyneuropathy condition starts from lower extremities which can progress to develop respiratory failure. Guillain-Barre syndrome (GBS) is the leading cause of acute flaccid paralysis in developed countries and is characterized by various degrees of weakness, sensory abnormalities and autonomic dysfunction. Although the underlying aetiology and pathophysiology of GBS are not completely understood, it is broadly believed that immune stimulation plays a role in its pathogenesis. Thus, since vaccines have an effect on the immune system it is biologically plausible that immunizations may be associated with subsequent GBS [5].

An immune response cross-reacts with peripheral nerve components because of molecular mimicry. The immune response can be directed towards the myelin or the axon of peripheral nerve, resulting in demyelinating and axonal forms of GBS. Campylobacter jejuni infection is the most commonly identified precipitant of GBS. Cytomegalovirus, Epstein-Barr virus, human immunodeficiency virus (HIV), and Zika virus have also been associated with GBS. A small percentage of patients develop GBS after another triggering event such as immunization, surgery, trauma, and bone-marrow transplantation [6]. Guillain-Barré syndrome (GBS) occurs world-wide with an overall incidence of 1 to 2 cases per 100,000 per year [7,8].
The initial diagnosis of Guillain-Barré syndrome (GBS) is based upon the clinical presentation. The cardinal clinical features of Guillain-Barré syndrome (GBS) are progressive, fairly symmetric muscle weakness accompanied by absent or depressed deep tendon reflexes. Patients usually present a few days to a week after onset of symptoms. The weakness can vary from mild difficulty with walking to nearly complete paralysis of all extremity, facial, respiratory, and bulbar muscles [6]. Severe respiratory muscle weakness necessitating ventilatory support develops in 10 to 30 percent [9]. GBS usually progresses over a period of about two weeks. By four weeks after the initial symptoms, >90 percent of GBS patients have reached the nadir of the disease [10].

In patients with GBS, lumbar puncture often reveals an elevated CSF protein with a normal CSF white blood cell count. This finding, known as albuminocytologic dissociation, is present in 50 to 66 percent of patients with GBS in the first week after the onset of symptoms and $\geq 75$ percent of patients in the third week [11]. A normal CSF protein is found in one-third to one-half of patients when tested earlier than one week after symptom onset and therefore does not exclude the diagnosis of GBS [12]. Since the nerve conduction abnormalities progress over time, serial electro diagnostic studies are frequently helpful [13]. Findings can be normal early in the course of GBS, and are typically most pronounced approximately two weeks after the onset of weakness [14].

\section{Conclusion}

To our knowledge there are only few cases reported with GBS syndrome accompanied with rhabdomyolysis. Our patient developed GBS following immunization and later complicated with rhabdomyolysis. This case emphasize the need to consider GBS in any patient who presents with weakness and muscle pain following vaccinations like HZV and PCV as in our case. Individuals with prior history of GBS may require particular attention before receiving vaccinations. It also focuses to closely monitor cpk levels to avoid development of fatal rhabdomyolysis. However, the benefit of vaccines in preventing disease and decreasing morbidity and mortality, particularly for influenza, needs to be weighed against the potential risk of GBS [5]. Flu vaccinations seem relatively safe [15].

\section{References}

1. Baxter R, Lewis N, Bakshi N, Vellozzi C, Klein NP (2012) CISA Network. Clin Infect Dis 54(6): 800-804.

2. Rokosz N, Rastawicki W, Jagielski M, Hetkowska-Abramczyk Z (2011) Med Dosw Mikrobiol 63(3): 255-261.

3. Ropper AH, Shahani BT (1984) Pain in Guillain-Barré syndrome. Arch Neurol 41(5): 511-514.

4. (2014) Indian J Crit Care Med 18(4): 241-243.

5. Haber P, Sejvar J, Mikaeloff Y, DeStefano F (2009) Vaccines and GuillainBarré syndrome. Drug Saf 32(4): 309-323.

6. www-up to date-com. Up to date 
7. Yuki N, Hartung HP (2012) Guillain-Barré syndrome. N Engl J Med 366: 2294-2304.

8. Sejvar JJ, Baughman AL, Wise M, Morgan OW (2011) Population incidence of Guillain-Barré syndrome: a systematic review and metaanalysis. Neuroepidemiology 36(2): 123-133.

9. Alshekhlee A, Hussain Z, Sultan B, Katirji B (2008) Guillain-Barré syndrome: incidence and mortality rates in US hospitals. Neurology 70(18): 1608-1613.

10. Fokke C, van den Berg B, Drenthen J, Walgaard C, van Doorn PA, et al. (2014) Diagnosis of Guillain-Barré syndrome and validation of Brighton criteria. Brain 137(Pt 1): 33-43.

11. Yuki N, Hartung HP (2012) Guillain-Barré syndrome. N Engl J Med 366: 2294-2309.
12. Fokke C, van den Berg B, Drenthen J, Walgaard C, van Doorn PA, et al. (2014) Diagnosis of Guillain-Barré syndrome and validation of Brighton criteria. Brain 137 (Pt 1): 33-43.

13. Uncini A, Kuwabara S (2012) Electrodiagnostic criteria for GuillainBarrè syndrome: a critical revision and the need for an update. Clin Neurophysiol 123(8): 1487-1495.

14. Hadden RD, Cornblath DR, Hughes RA, Zielasek J, Hartung HP, et al. (1998) Electrophysiological classification of Guillain-Barré syndrome: clinical associations and outcome. Plasma Exchange/Sandoglobulin Guillain-Barré Syndrome Trial Group. Ann Neurol 44(5): 780-788.

15. Kuitwaard K, Bos-Eyssen ME, Blomkwist-Markens PH, van Doorn PA (2009) Recurrences, vaccinations and long-term symptoms in GBS and CIDP. J Peripher Nerv Syst 14(4): 310-315.

\section{Your next submission with Juniper Publishers} will reach you the below assets

- Quality Editorial service

- Swift Peer Review

- Reprints availability

- E-prints Service

- Manuscript Podcast for convenient understanding

- Global attainment for your research

- Manuscript accessibility in different formats

( Pdf, E-pub, Full Text, Audio)

- Unceasing customer service

Track the below URL for one-step submission https://juniperpublishers.com/online-submission.php 